\title{
Temporal Logics of Agency
}

\author{
Johan van Benthem • Eric Pacuit
}

Published online: 9 January 2010

(C) The Author(s) 2010. This article is published with open access at Springerlink.com

Time is the grand stage where human activities take place (rational or otherwise). And the view of a branching temporal universe, or tree of possible events, with our actual history linearly advancing through it, is a widely shared cultural idea, not confined to Academia (cf. Borges brilliant 1941 essay El Jardín de senderos que se bifurcan). Even though logic is often considered a study of timeless propositions, temporal languages and logics over tree structures have a long tradition. Philosophers have studied temporal structure and temporal reasoning since the 1950s, from the 'tense logic' of Prior (1967) to the 'STIT' system of Belnap et al. (2001). Moreover, starting from 1970s, computer scientists have joined in, and developed many further flavours of temporal logic, with major strands such as Pnueli on program correctness (Manna and Pnueli 1991), Emerson and Clarke on process specification and verification (Emerson and Clarke 1980, 1982), Reiter on the situation calculus in AI (Reiter 2001), and Thomas on the automata-theoretic foundations of computing (Thomas 1990). In addition, the pure first- and second-order logic of tree-like structures, starting from Rabin's classic decidability result (Rabin 1969), provides deeper background (cf. Grädel et al. (2002)). The chapter by Hodkinson and Reynolds on 'Temporal Logic' in the Handbook of Modal Logic (Hodkinson and Reynolds 2006) brings together many of these trends in one mathematical narrative. But the field of reasoning in, and about, time can be mapped out in many further ways: Van Benthems chapter 'Temporal Logic' in the

\footnotetext{
J. van Benthem $(\varangle)$

University of Asmterdam, Amsterdam, The Netherlands

e-mail: johan.vanbenthem@uva.nl

J. van Benthem

Stanford University, Stanford, CA, USA

E. Pacuit

Center for Logic and Philosophy of Science, Tilburg University, Tilburg, The Netherlands

e-mail: E.J.Pacuit@uvt.nl
} 
Handbook of Logic in Artificial Intelligence and Logic Programming (van Benthem 1995) also includes issues in natural language semantics, discourse representation, and the duality between interval and point ontologies.

Nowadays, temporal logics play an important role in theories of agency, an area where philosophy and computer science meet with other disciplines, such as game theory. Over the last decades, various paradigms have emerged for studying agents that process information and acquire knowledge while acting over time. In computer science, Interpreted Systems (Fagin et al. 1995) and Epistemic Temporal Logic (Parikh and Ramanujam 1985, 2003) are major examples, while a major philosophical paradigm for agents deliberating and deciding over time is STIT (Belnap et al. 2001). As one refines the study of agents, still further attitudes come into play, such as their beliefs, preferences, and their strategies for interaction. Indeed, finite and infinite extensive games as long studied in game theory (Osborne and Rubinstein 1994) fall into this category as well. Tree-like structures again form a natural Grand Stage for the formal logical study of all these more complex agent-related dynamic processes. One can see modern theories of agency as merging ideas from philosophical logic and computational logic, thereby crossing traditional boundaries in content and style.

In our view, all these paradigms are after similar goals, and in doing so, they show striking similarities. Think of a historical analogy: in the 1930s, many different paradigms addressed the nature of computation, and what emerged, as stated explicitly in Church's Thesis, was a unified view of congenial, often equivalent perspectives on computation, an integrated theory where insights about one framework can transfer to another. This diversity plus underlying unity of frameworks is still an asset today in fine-tuning models of concrete computational phenomena. The area of agency may be viewed as a rich modern successor to computation, and we feel that it might be heading the same way. Admittedly, there are many churches and sects with a religious following, and occasional outbursts of sniping occur. But still, we see many suggestive indications of peaceful convergence. Here is one promising sign: insights can cross over. For instance, the results in Halpern and Vardi (1989) about unexpected complexity effects of apparently harmless assumptions about epistemic agents such as Perfect Recall, though developed inside the paradigm of Interpreted Systems, have turned out widely applicable (cf. Gabbay et al. 2003; Kurucz 2006). And likewise, our own research has found strong analogies between epistemic temporal logic and, at a first glance, quite different 'dynamic epistemic logics' that focus on the detailed process structure of single informational acts and events (see the representation theorems and combined dynamic-temporal logics in van Benthem and Pacuit (2006), van Benthem et al. (2009) and Hoshi's contribution to this special issue). Moreover, these studies show that different temporal frameworks for agency can merge to produce new research questions on shared challenges like finding optimal logics of plans or protocols, or studying processes of belief merge, group creation and collective action.

The handbook chapters that we mentioned above do a valuable job in bringing together and comparing perspectives. Also, new journals like "Knowledge, Rationality and Action" do a great job in merging communities and creating common knowledge of existing relevant achievements in mathematical logic, foundations of computing, artificial intelligence, philosophy, or game theory. This issue of JoLLI is a modest step toward consolidating the technical foundations of all this. It started from a 2006 
Workshop on "Temporal Logics of Agency" where we gathered a variety of congenial speakers. ${ }^{1}$ Building on the success of that event we sought contributions that demonstrate a broad set of bridges between different temporal logic paradigms that belong together in our view. We selected 5 papers for this special issue that highlight the interesting issues that arise when different logical systems for agency are compared and their ideas are merged:

1. Information Tracking in Games on Graphs by Dietmar Berwanger and Łukasz Kaiser

2. Merging DEL and ETL by Tomohiro Hoshi

3. The Situational Calculus: A Case for Modal Logic by Gerhard Lakemeyer

4. Automata for epistemic temporal logic with synchronous communication by Swarup Mohalik and R. Ramanujam.

5. Combinations of Stit and Actions by Ming $\mathrm{Xu}$

These contributions can be very roughly divided into two categories:

Comparing logical frameworks: Sometimes differences between logical frameworks that model similar aspects of social interaction reflect the mathematical conventions used in different research communities. A certain amount of technical work can wipe away the differences and allow results to transfer (for contributions along these lines, see Pacuit (2007), Lomuscio and Ryan (1997), Halpern (1999), Goranko and Jamroga (2004)). Other differences may point to important foundational issues, such as the classic debate about "branching-time" versus "linear-time" temporal logics in the model checking literature (see, for example, Emerson and Halpern (1986)). The contributions in this volume highlight important conceptual issues that arise when confronting different styles of modeling rational agents interacting over time. Lakemeyer provides an insightful comparison between different logical systems for reasoning about knowledge and time: the Situation Calculus and modal logic. Hoshi's contribution contrasts the two major paradigms for reasoning about information change (epistemic temporal logic and dynamic epistemic logic) showing that they focus on important different aspects of social interaction (global 'protocol information' in the first case and local 'epistemic dynamics' in the second case). Berwanger and Kaiser bring together perspectives from automata-based games in computer science and issues of imperfect information and prediction that stem from game theory. Finally, Ming Xu offers a much needed comparison between two major perspectives on modeling actions: the "state-change" view found in epistemic temporal logics and dynamic epistemic logics, and the "agency" view restricting future histories found in the stit logics.

Merging ideas: But our authors also go on and create new combinations of different frameworks. Lakemeyer shows how modal viewpoints can naturally complement the Situation Calculus, and proposes a first-order modal situation calculus that combines

\footnotetext{
1 The workshop Converging Paradigms? Comparing Temporal Frameworks for Logics of Knowledge, Belief, Action, and Choice took place at the Institute for Logic, Language and Computation at the University of Amsterdam on December 19, 2006. More information on the event can be found at ai.stanford.edu/ epacuit/socsit.
} 
virtues of both frameworks. Hoshi studies logics that combine the representational power of dynamic epistemic logics with the explicit protocols of epistemic temporal logics, giving a much richly layered view of informational processes. Xu shows how the explicit actions of epistemic temporal logics can be represented in the powerful stit logics of agency, replacing competition by cooperation. But we are not only interested in combining logical systems, but also more generally, merging ideas from different communities. Mohalik and Ramanujam develop the automata-theoretic underpinnings of epistemic temporal logics, providing much richer accounts of agents. Finally, Berwanger and Kaiser use key ideas from game theory and automata theory to offer a fascinating new 'tracking' perspective on finitely representable infinite interactive processes.

We feel that the papers in this issue provide a lively and useful picture of current logical research into agency over time. Perhaps this meeting is already of importance in itself, as too little is often known across communities, and one has to start with small things. In terms of knowledge merge, in our own comparative work, we have found that even just recognizing the same result in another framework can be a creative event. But as we said, a true grasp of temporal logic today requires merging insights from mathematical logic, philosophical logic, computational logic, and logics of games. But, as we have also said, such confluences remain auxiliary goals toward a still grander goal (van Benthem 2009): the logical study of agency as one coherent phenomenon.

Whether or not our authors share this final grand ambition, their contributions also stand on their own. We thank all of them for their smooth and pleasant cooperation, and we hope that the reader will profit as much from reading their papers as we did.

Open Access This article is distributed under the terms of the Creative Commons Attribution Noncommercial License which permits any noncommercial use, distribution, and reproduction in any medium, provided the original author(s) and source are credited.

\section{References}

Belnap, N., Perloff, M., \& Xu, M. (2001). Facing the future: Agents and choice in our indeterminist world. Oxford: Oxford University Press.

Blackburn, P., van Benthem, J., \& Wolter, F. (Eds.). (2007). Handbook of modal logic. Amsterdam: Elsevier.

Emerson, E. A., \& Clarke, E. M. (1980). Characterizing correctness properties of parallel programs using fixpoints. In: Automata, Languages and Programming, Lecture Notes in Computer Science (Vol. 85, pp. 169-181). Springer.

Emerson, E. A., \& Clarke, E. M. (1982). Using branching time temporal logic to synthesize synchronization skeletons. Science of Computer Programming, 2(3), 241-266.

Emerson, E. A., \& Halpern, J. (1986). sometimes and not never revisited: On branching versus linear time temporal logic. Journal of the ACM, 33(1), 151-178.

Fagin, R., Halpern, J., Moses, Y., \& Vardi, M. (1995). Reasoning about Knowledge. Boston: The MIT Press.

Gabbay, D., Hogger, C. J., \& Robinson, J. A. (Eds.). (1993). Handbook of logic in artificial intelligence and logic programming: Volume 4, epistemic and temporal reasoning. Oxford: Oxford University Press.

Gabbay, D., Kurucz, A., Wolter, F., \& Zakharyaschev, M. (2003). Many-dimensional modal logics: Theory and applications. Amsterdam: Elsevier.

Goranko, V., \& Jamroga, W. (2004). Comparing semantics of logics for multi-agent systems. Synthese: Knowledge, Rationality, and Action, 139(2), 241-280. 
Grädel, E., Thomas, W., \& Wilke, T. (Eds.) (2002). Automata, logics and infinite games: A guide to current research. Lecture Notes in Computer Science (Vol. 2500). Springer.

Halpern, J. (1999). Set-theoretic completeness for epistemic and conditional logic. Annals of Mathematics and Artificial Intelligence, 26, 1-27.

Halpern, J., \& Vardi, M. (1989). The complexity of reasoning about knowledge and time. Journal of Computer and System Sciences, 38, 195-237.

Hodkinson, I., \& Reynolds, M. (2006). Temporal logic. In: Handbook of modal logic (Blackburn, van Benthem and Wolter 2007), pp. 655-720. Amsterdam: Elsevier.

Kurucz, A. (2006). Combining modal logics. In: Handbook of Modal Logic (Blackburn, van Benthem and Wolter 2007), pp. 869-924. Amsterdam: Elsevier.

Lomuscio, A., \& Ryan, M. (1997). On the relation between interpreted systems and Kripke models. In: Proceedings of the AI97 Workshop on Theoretical and Practical Foundation of Intelligent Agents and Agent-Oriented Systems (Vol. LNCS 1441, pp. 46-59).

Manna, Z., \& Pnueli, A. (1991). The temporal logic of reactive and concurrent systems: Specification. New York: Springer.

Osborne, M., \& Rubinstein, A. (1994). A course in game theory. Cambridge: MIT Press.

Pacuit, E. (2007). Some comments on history based structures. Journal of Applied Logic, 5(4), 613-624.

Parikh, R., \& Ramanujam, R. (1985). Distributed processes and the logic of knowledge. In: Logic of Programs, Lecture Notes in Computer Science (vol. 193, pp. 256-268). Springer.

Parikh, R., \& Ramanujam, R. (2003). A knowledge based semantics of messages. Journal of Logic, Language and Information, 12, 453-467.

Prior, A. (1967). Past, present and future. Oxford: Oxford University Press.

Rabin, M. (1969). Decidability of second-order theories and automata on infinite trees. Transactions of the American Mathematical Society, 141, 1-35.

Reiter, R. (2001). Knowledge in action: Logical foundations for specifying and implementing dynamic systems. Cambridge: The MIT Press.

Thomas, W. (1990). Automata on infinite objects. In J. van Leeuwen (Ed.), Handbook of theoretical science: Volume B, formal models and semantics (pp. 133-192). Amsterdam: Elsevier.

van Benthem, J. (1995). Temporal logic. In: Handbook of logic in artificial intelligence and logic programming: Volume 4, epistemic and temporal reasoning (Gabbay et al. 1993), pp. 241-350. Oxford University Press.

van Benthem, J. (2009). Logical dynamics of information and interaction, Book manuscript.

van Benthem, J., Gerbrandy, J., Hoshi, T., \& Pacuit, E. (2009). Merging frameworks for interaction. Journal of Philosophical Logic, 38(5), 491-526.

van Benthem, J., \& Pacuit, E.(2006). The tree of knowledge in action: Towards a common perspective. In: G. Governatori, I. Hodkinson \& Y. Venema (Eds.), Proceedings of advances in modal logic (Vol. 6, pp. 87-106). London: King's College Press. 